\title{
Yield of tuberculosis screening in asylum-seekers by country of origin: analysis of screening data in a German federal state (2002-2015)
}

\begin{abstract}
To the Editor:
Upon-entry screening for tuberculosis (TB) in immigrants plays a major role in national TB programmes of many low TB incidence countries [1]. The majority of European countries conduct routine screening for active TB in refugees and asylum-seekers using chest radiography alone or combined with other diagnostics [2]. Few countries, however, perform targeted screening among the heterogeneous group of asylum-seekers and refugees by using specific selection criteria [3]. Country of origin may serve as a selection criterion [4] to target and prioritise specific sub-groups and avoid "indiscriminate mass screening" [5]. Targeted screening would support front-line health workers to cope with the challenge of performing screening for TB in times of high immigration [6], e.g. in Germany when about 1 million new asylum-seekers were registered in 2015. Despite being desirable, so far a lack of empirical evidence precludes the (objective) determination of a suitable target group in asylum-seekers and refugees: only a few studies have reported the yield of screening for TB by country of origin [7].
\end{abstract}

We analysed the yield of active TB (per 100000 asylum-seekers) and the number needed to screen (NNS) to identify one case (1/yield) [5], stratified by age-group, sex and country of origin using data of 119037 asylum-seekers from $>80$ countries undergoing mandatory upon-entry screening in a state reception centre (2002-2015) in South Germany (Karlsruhe, Baden-Württemberg). The centre quasi-randomly received about $13 \%$ of newly arriving asylum-seekers to Germany based on a quota (Königsteiner Schlüssel). Newly arriving asylum-seekers reside in shared accommodation facilities and must undergo a mandatory health examination according to national law (Asylum Law and Infection Protection Act). The health examination consists of symptom-based screening (all asylum-seekers) and a compulsory chest radiograph (asylum-seekers 15 years and older except pregnant women) to identify pulmonary TB [8]. Upon-entry screening for TB in children or pregnant women is governed by different policies at the level of the 16 federal states $[8,9]$. In the state of Baden-Württemberg, the initial screening consisted of a tuberculin skin test (2002-2010) and symptom-based screening (2011-2015) for children $<15$ years, and a tuberculin skin test or interferon- $\gamma$ release assay for pregnant women in the observation period [8]. Asylum-seekers with presumptive TB undergo further diagnosis by means of a facultative chest computed tomography scan, a sputum test (microscopy or PCR) and culture tests. In this study a TB case was defined by the fact that the physician decided to start treatment (including clinically diagnosed cases, smear-positive cases and/or culturally confirmed TB). According to German law, the local public health authority must be notified about these cases. The results of the health entry examination, including the final results of mandatory $\mathrm{TB}$ screening, are registered by the local public health authority in an electronic self-designed database. As the study findings were based on anonymous secondary data, the study was exempt from ethical clearance according to the regulations of the medical ethics committee of the Medical Faculty of Heidelberg University.

The country of origin was determined by the asylum-seekers' nationality. We focused on country of origin as a predictor variable, as opposed to a classification of countries, e.g. based on their TB incidence, since we were ultimately interested in country-specific effects and predictions. Not all individual country effects could be estimated since the ability to fit a model depends on the number of detected cases and the

@ERSpublications

Yield of TB screening varies by country of origin; targeted approaches are needed to increase screening efficiency http://ow.ly/SuvZ30941Ej

Cite this article as: Bozorgmehr K, Joggerst B, Wagner $\mathrm{U}$, et al. Yield of tuberculosis screening in asylumseekers by country of origin: analysis of screening data in a German federal state (2002-2015). Eur Respir J 2017; 49: 1602327 [https://doi.org/10.1183/13993003.02327-2016]. 
number of observations, and reasonably precise estimates could only be expected from countries with either substantial case load or screening volume (or, at best, both). We thus denoted countries with five or more detected active TB cases as "high-case" and those with $>5000$ individuals screened as "high-screen" countries. Crude and age-/sex-adjusted relative risks (RRs) of TB with 95\% credible intervals (CrIs) were obtained in each group from Bayesian Poisson regression models. The analyses were performed using "Stan" [10] via the interface "rstan" in the R language and environment for statistical computing.

A total of 98 cases of active TB were identified in the scope of upon-entry screening in 119037 asylum-seekers (mean \pm SD age, $25 \pm 13$ years; 69\% male) from more than 80 countries over 14 years, corresponding to an overall yield of 82 (per 100000) and an overall NNS of $1215.69 \%$ of cases were detected in asylum-seekers from nine different countries of origin (Cameroon, Eritrea, Gambia, Georgia, Kosovo, Pakistan, Russia, Somalia and Syria). Absolute numbers of identified cases per year increased substantially after 2013, but the overall yield decreased by more than $50 \%$ in 2015 compared with the preceding decade (2002-2012), indicating a decline in screening efficiency because of the changing composition of the screened population. The yield was highest in asylum-seekers aged 20-39 years. The estimated NNS was highest for children $<15$ years and women, and ranged between 146 for asylum-seekers from Somalia (lowest) to 9955 for asylum-seekers from Iraq (highest) (table 1).

The yield of screening in asylum-seekers from the majority of countries was below 200 per 100000 (except Cameroon, Georgia, Gambia, Sudan, Vietnam, Eritrea and Somalia). More than 10000 asylum-seekers from Iraq, Syria and Kosovo were screened, with a yield of $<50$ per 100000 . The adjusted RR for countries in the high-case group relative to other countries ranged from 11.85 (95\% CrI 4.70-26.65) for Somali to $0.67(0.22-1.68)$ for Syrian asylum-seekers. For countries in the high-screen group it ranged from $1.78(0.99-3.15)$ for Gambia to $0.06(<0.01-0.36)$ for Iraq.

Few studies have yet analysed the yield of TB screening in asylum-seekers by country of origin adjusted for age and sex [7]. Our analysis suggests that active TB cases identified by upon-entry screening are concentrated in a few countries, and that efficiency of screening is particularly high and therefore more warranted in these countries (e.g. Cameroon, Eritrea, Gambia, Georgia, Pakistan, Russia and Somalia). Based on an analysis of national data, the Netherlands also observed a decline in annual yield of screening because of high immigration of asylum-seekers from countries with traditionally low TB incidence such as Iraq, Iran and Syria. Screening in asylum-seekers with a TB incidence in the country of origin $<50$ per 100 000 population (such as Syria) was therefore stopped, and an NNS of 2000 was used as a cut-off to decide on the continuation of screening [11].

Based on this cut-off and the estimated NNS in our study, screening by chest radiograph in asylum-seekers from Afghanistan, Iraq, Kosovo, Macedonia and Syria would need to be discontinued and replaced by other case-finding activities. However, TB is a rare outcome and previous country-specific analyses are based on a few cases [11] or on aggregated continent data [7]. This entails high uncertainty (i.e. wide CrIs) in estimates of yield and NNS. The estimated NNS for some countries in our sample, especially "high-screen" countries such as Iraq, Afghanistan, Syria, Macedonia and Kosovo, is very high, but the CrIs are wide and often include the cut-off of 2000. The NNS estimates for the "high-case" countries were more precise. Continuing screening in asylum-seekers from "high case" countries is likely to increase efficiency and appears to be "a good bet", but discontinuing screening in the other group would come with considerable uncertainty. This uncertainty could be reduced, for example by pooling data across regions and countries with comparable screening protocols [12], and/or by using external (prior) information, e.g. from WHO prevalence data or clinical assessments [13] in order to obtain better estimates of country-specific risk. Uncertainty aside, the current practice of (indiscriminate) screening for TB in the heterogeneous group of asylum-seekers appears to have potential for optimising efficiency. Our analysis thus reconfirms overall concerns about the effectiveness [1,5] and costs [14] of screening. Estimates of average yield of TB screening in asylum-seekers, such as those generated by systematic reviews [1] and meta-analyses [7, 15] are indicative, but are at the same time only crude estimates masking substantial variance in yield among asylum-seekers from different countries of origin.

Characteristics such as sex and age are also likely to affect the yield of screening. The NNS among children aged $<15$ years was very high and above the cut-off of 2000 . Only four cases of TB in children were identified (2002-2015), and it cannot be ruled out that these were identified primarily because of contact investigations of infected relatives. Further research is necessary to assess the effectiveness in this vulnerable sub-group of asylum-seekers, as country of origin may play a role among these as well. Timing of screening, transmissions avoided or the number of false negatives would be relevant areas to consider in future studies.

The study underlines the need for more targeted TB screening strategies. To this end, external (prior) information and large data samples gained by pooling datasets are needed to derive precise estimates of the risk for TB in asylum-seekers by country of origin. 
TABLE 1 Yield of screening, number needed to screen, and relative rates of yield obtained from Bayesian Poisson regression models

\begin{tabular}{|c|c|c|c|c|c|c|}
\hline \multirow[t]{2}{*}{ Characteristic } & \multirow[t]{2}{*}{$\mathbf{N}$} & \multirow{2}{*}{$\begin{array}{l}\text { Cases with active } \\
\text { TB detected }\end{array}$} & \multirow{2}{*}{$\begin{array}{l}\text { Yield of active TB per } \\
100000 \text { screened }(95 \% \text { Crl) }\end{array}$} & \multirow{2}{*}{$\begin{array}{l}\text { Number needed to } \\
\text { screen to detect } 1 \text { case } \\
\text { of active TB }(95 \% \text { Crl) }\end{array}$} & \multicolumn{2}{|c|}{ Relative risk ( $95 \%$ CrI) } \\
\hline & & & & & $\begin{array}{c}\text { Mutually adjusted model I: } \\
\text { countries }>5 \text { cases versus other }\end{array}$ & $\begin{array}{l}\text { Mutually adjusted model II: countries } \\
\text { with }>5000 \text { screened versus other }\end{array}$ \\
\hline \multicolumn{7}{|l|}{ Age group } \\
\hline$<15$ years & 24249 & 4 & $16(6,39)$ & $6062(2550,17959)$ & $0.20(0.08,0.64)$ & $0.22(0.07,0.59)$ \\
\hline $15-24$ years & 35390 & 35 & $99(70,136)$ & $1011(736,1427)$ & $0.74(0.48,1.16)$ & $0.85(0.55,1.31)$ \\
\hline $25-44$ years & 51111 & 56 & $110(84,141)$ & $913(708,1196)$ & Ref. & Ref. \\
\hline $45-64$ years & 7583 & 3 & $40(11,106)$ & $2528(947,8974)$ & $0.48(0.13,1.42)$ & $0.45(0.11,1.31)$ \\
\hline$>64$ years & 616 & 0 & & & $0.06(<0.01,3.11)$ & $0.03(1.18,3.94)$ \\
\hline \multicolumn{7}{|l|}{ Sex } \\
\hline Female & 36872 & 13 & $35(20,59)$ & $2836(1707,5060)$ & Ref. & Ref. \\
\hline Male & 82051 & 85 & $104(83,127)$ & $965(785,1200)$ & $2.09(1.17,3.97)$ & $2.09(1.15,3.96)$ \\
\hline \multicolumn{7}{|l|}{ Year } \\
\hline $2002-2012$ & 36647 & 41 & $112(81,150)$ & $894(666,1228)$ & Ref. & Ref. \\
\hline 2013 & 14407 & 7 & $49(22,95)$ & $2058(1048,4601)$ & $0.42(0.17,0.91)$ & $0.48(0.20,1.05)$ \\
\hline 2014 & 23916 & 24 & $100(66,147)$ & $997(681,1516)$ & $0.65(0.36,1.17)$ & $0.99(0.58,1.68)$ \\
\hline 2015 & 44067 & 26 & $59(39,85)$ & $1695(1175,2534)$ & $0.52(0.30,0.90)$ & $0.68(0.40,1.14)$ \\
\hline \multicolumn{7}{|l|}{ Country\# } \\
\hline Afghanistan & 7433 & 2 & $27(6,86)$ & $3716(1159,17883)$ & & $0.22(0.04,0.82)$ \\
\hline Cameroon & 2506 & 5 & $200(76,437)$ & $501(229,1313)$ & $2.70(0.90,6.68)$ & \\
\hline Eritrea & 1941 & 9 & $464(230,845)$ & $216(118,435)$ & $8.95(3.83,19.77)$ & \\
\hline Gambia & 6971 & 18 & $258(159,399)$ & $387(251,630)$ & $4.53(2.42,8.37)$ & $1.78(0.99,3.15)$ \\
\hline Georgia & 2121 & 5 & $236(90,516)$ & $424(194,1111)$ & $4.05(1.37,10.26)$ & \\
\hline Iraq & 9955 & 1 & $10(1,47)$ & $9955(2130,92262)$ & & $0.06(<0.01,0.36)$ \\
\hline Kosovo & 20336 & 7 & $34(15,68)$ & $2905(1480,6494)$ & $0.86(0.34,1.91)$ & $0.37(0.15,0.80)$ \\
\hline Macedonia & 7005 & 2 & $29(6,92)$ & $3502(1092,16854)$ & & $0.31(0.06,1.14)$ \\
\hline Pakistan & 5103 & 7 & $137(61,269)$ & $729(372,1629)$ & $1.88(0.74,4.17)$ & $0.87(0.37,1.84)$ \\
\hline Russia & 3026 & 5 & $165(63,362)$ & $605(276,1585)$ & $3.17(1.07,7.90)$ & \\
\hline Somalia & 1025 & 7 & $683(306,1336)$ & $146(75,327)$ & $11.85(4.70,26.65)$ & \\
\hline Syria & 17172 & 5 & $29(11,64)$ & $3434(1567,9000)$ & $0.67(0.22,1.68)$ & $0.27(0.09,0.65)$ \\
\hline Other & & & & & Ref. & Ref. \\
\hline
\end{tabular}

The Watanabe-Akaike information criterion (WAIC) was 304 in the mutually adjusted model I and 260 in the mutually adjusted model II. Lower values of the WAIC indicate a better prediction model. \#: countries with either $\geqslant 5$ cases of active tuberculosis (TB) detected or more than 5000 individuals screened. RR: relative risk; Crl: credible interval. 
Kayvan Bozorgmehr ${ }^{1}$, Brigitte Joggerst ${ }^{2}$, Ulrich Wagner ${ }^{3}$ and Christian Stock ${ }^{4}$

${ }^{1}$ Dept of General Practice and Health Services Research, University Hospital Heidelberg, Heidelberg, Germany. ${ }^{2}$ Public Health Authority, Pforzheim, Germany. ${ }^{3}$ Public Health Authority, Section for Disease Control, Karlsruhe, Germany. ${ }^{4}$ Institute of Medical Biometry and Informatics, University Hospital Heidelberg, Heidelberg, Germany.

Correspondence: Kayvan Bozorgmehr, Dept of General Practice and Health Services Research, University Hospital Heidelberg, Marsilius Arkaden, INF 130.2, 69120 Heidelberg, Germany. Email: kayvan.bozorgmehr@med.uni-heidelberg.de

Received: Oct 142016 | Accepted after revision: Dec 262016

Support statement: This study was part of the BMBF-funded project RESPOND ("Improving Regional Health System Responses to the challenge of forced migration through tailored interventions for asylum-seekers and refugees"). The study received financial support by the Federal Ministry of Research and Education (BMBF) in the scope of the research programme "Strukturaufbau in der Versorgungsforschung" (grant number: FKZ 01GY1611). Funding information for this article has been deposited with the Crossref Funder Registry.

Conflict of interest: Disclosures can be found alongside this article at erj.ersjournals.com

Acknowledgements: The authors thank Ursula Paschmann (Public Health Services, Section for Disease Control, Landkreis Karlsruhe, Germany) for support in data collection and data management.

Authors' contributions: conceived the study: K. Bozorgmehr, B. Joggerst and C. Stock. Data collection: B. Joggerst and U. Wagner. Data analysis: C. Stock. First and last version of manuscript: Kayvan Bozorgmehr. All authors made important contributions to the study design, participated in drafting the article and revising it critically for important intellectual content, and gave final approval of the version to be submitted.

\section{References}

1 Klinkenberg E, Manissero D, Semenza JC, et al. Migrant tuberculosis screening in the EU/EEA: yield, coverage and limitations. Eur Respir J 2009; 34: 1180-1189.

2 Dara M, Solovic I, Sotgiu G, et al. Tuberculosis care among refugees arriving in Europe: a ERS/WHO Europe Region survey of current practices. Eur Respir J 2016; 48: 808-817.

3 Pareek M, Baussano I, Abubakar I, et al. Evaluation of immigrant tuberculosis screening in industrialized countries. Emerg Infect Dis 2012; 18: 1422-1429.

4 Erkens C, Slump E, Kamphorst M, et al. Coverage and yield of entry and follow-up screening for tuberculosis among new immigrants. Eur Respir J 2008; 32: 153-161.

5 WHO. Systematic screening for active tuberculosis: Principles and recommendations. Geneva, World Health Organization, 2013.

6 Akkerman OW, de Lange WC, Schölvinck EH, et al. Implementing tuberculosis entry screening for asylum seekers: the Groningen experience. Eur Respir J 2016; 48: 261-264.

7 Arshad S, Bavan L, Gajari K, et al. Active screening at entry for tuberculosis among new immigrants: a systematic review and meta-analysis. Eur Respir J 2010; 35: 1336-1345.

8 Wahedi K, Nöst S, Bozorgmehr K. [Health examination of asylum seekers: A nationwide analysis of state policies in Germany: $₫ 62$ of the asylum law]. Bundesgesundheitsblatt Gesundheitsforschung Gesundheitsschutz 2016; 60: 108-117.

9 Bozorgmehr K, Nöst S, Thaiss MH, et al. [Health care provisions for asylum-seekers: a nationwide survey of public health authorities in Germany]. Bundesgesundheitsblatt Gesundheitsforschung Gesundheitsschutz 2016; 59: 545-555.

10 Carpenter B, Gelman A, Hoffman M, et al. Stan: A probabilistic programming language. J Stat Softw 2016; 55: 1-37.

11 de Vries G, van Rest J, Meijer W, et al. Low yield of screening asylum seekers from countries with a tuberculosis incidence of $<50$ per 100000 population. Eur Respir J 2016; 47: 1870-1872.

12 de Vries G, van Rest J, Meijer W, et al. Tuberculosis screening yield of asylum seekers in Europe. Eur Respir J 2016; 48: 1255-1256.

13 Dara M, Solovic I, Goletti D, et al. Preventing and controlling tuberculosis among refugees in Europe: more is needed. Eur Respir J 2016; 48: 272-274.

14 Dasgupta K, Menzies D. Cost-effectiveness of tuberculosis control strategies among immigrants and refugees. Eur Respir J 2005; 25: 1107-1116.

15 Bozorgmehr K, Razum O, Saure D, et al. Yield of active screening for tuberculosis among asylum-seekers in Germany: a systematic review and meta-analysis. Euro Surveill 2017; in press. 